\title{
Fast microwave assisted synthesis of $p$-methoxyphenyltellurium trichloride
}

\author{
Julio A. Seijas,* M. Pilar Vázquez-Tato,* Alberto Mena-Menéndez \\ Departamento de Química Orgánica. Facultade de Ciencias. Universidade de Santiago de \\ Compostela. Campus de Lugo. Alfonso X el Sabio, 27002-Lugo. Spain
}

Abstract: Microwave assisted formation of C-Te bonds is applied to the preparation of p-methoxyphenyltellurium trichloride in a fast, direct and simple way.

Aryl chalcogenides structural motifs are commonly found in a variety of molecules with biological interest ${ }^{1}$ and new materials ${ }^{2}$. Furthermore, organotellurium compounds have been used as precursors in the synthesis of molecules with different functionalities, such as dienes and enediynes, ${ }^{3}$ present in the structure of important classes of natural products. $^{4}$

This communication deals with the synthesis of aryltellurium trihalides $\operatorname{ArTeX}_{3}$. These can be used in the arylation of olefins ${ }^{5}$ and in the preparation of several organotellurium derivatives $^{6}$ and have been identified as biocides. ${ }^{7}$

Up to date, few studies have been devoted to the use of microwave irradiation to enhance tellurium chemistry either with $^{8}$ or without ${ }^{9}$ formation of C-Te bonds. Among the several methods ${ }^{6}$ available for the synthesis of $\mathrm{ArTeX}_{3}$ we chose the condensation of tellurium tetrachloride with aromatic compounds, in order to study the possibility of enhancing the reaction by microwave irradiation.

The first attempt, based on Comasseto's synthesis by conventional heating, ${ }^{10}$ to get $p$ methoxyphenyltellurium trichloride from anisole (1) and tellurium tetrachloride was done by irradiating a mixture of both reagents at $120^{\circ} \mathrm{C}$ under solvent-free conditions. However, only decomposition of the reaction mixture was achieved. Thus, the reaction was checked in the presence of solvent. ${ }^{11}$ The irradiation for 15 minutes of a suspension of anisole and $\mathrm{TeCl}_{4}$ in refluxing carbon tetrachloride did not lead to an appreciable amount of 2 .

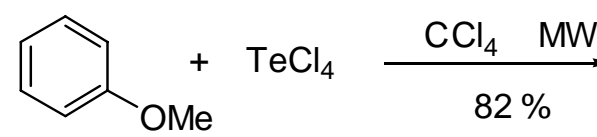

1<smiles>COc1ccc([Te](Cl)(Cl)Cl)cc1</smiles>

2

\section{Scheme 1}


With the purpose of using higher temperatures the irradiation was carried out in a sealed vessel. Hence, a 1:1 ratio of anisole and $\mathrm{TeCl}_{4}$ was irradiated for 10 minutes with the maximum temperature control set at $120^{\circ} \mathrm{C}$ and irradiation power $80 \mathrm{~W}$, yielding a $73 \%$ of $p$-methoxyphenyltellurium trichloride (2). Raising the irradiation power to $100 \mathrm{~W}$ in the same reaction time the yield reached $82 \%$, higher irradiation powers (200W, 250W) did not increase the yield.

In summary, here is reported the first microwave-assisted formation of aryltellurides. This proves that microwaves are a valuable tool for the formation of Ar-C bonds, opening a new facet in the field of organochalcogenide chemistry.

\section{Acknowledgements}

XUNTA DE GALICIA for financial support: PGIDIT05PXIB26201PR.

\section{Experimental procedure}

Anisole (540 mg, $5 \mathrm{mmol}$ ), $\mathrm{TeCl}_{4}(135 \mathrm{mg}, 0,5 \mathrm{mmol})$ and $\mathrm{CCl}_{4}$ are mixed in a sealed vessel. Reaction mixture was irradiated in a CEM Discover monomode oven for 10 minutes at $120^{\circ} \mathrm{C}(100 \mathrm{~W})$. On cooling the reaction mixture, a yellow crystalline solid crystallizes out, this was filtered and vacuum dried. The solid (139 mg, 82\%) was identified as p-methoxyphenyltellurium trichloride (2). IR(Golden-Gate): 3013, 1566, 1485, 1256, 1180, 1046, $817 \mathrm{~cm}^{-1} .{ }^{1} \mathrm{H}$ NMR $\left(\mathrm{CDCl}_{3}\right) \delta 3.81$ (s, 3H, $\left.\mathrm{OCH}_{3}\right), 6.99(\mathrm{~d}$, 2H, J=9Hz.), 8.31 (d, 2H, $J=9 \mathrm{~Hz}.) .{ }^{13} \mathrm{C} \mathrm{NMR}\left(\mathrm{CDCl}_{3}\right) \delta 56.16,114.06,114.64$, 134.59, 135.93. MS m/z (\%) $344\left(\mathrm{M}^{+}+4,39\right), 342\left(\mathrm{M}^{+}+2,36\right), 340\left(\mathrm{M}^{+}\left[3 \times \mathrm{Cl}_{35}, \mathrm{Te}_{128}\right]\right.$, 23), 307 (19), 305 (15), 303 (8), 272 (85), 270 (73), 268 (40), 237 (65), 235 (60), 233 (37), 214 (100), 199 (87), 142 (90).

\footnotetext{
${ }^{1}$ Kaldor, S. W.; Kalish, V. J.;Davies II, J. F.; Shetty, B. V.; Fritz, J. E.; Appelt, K.; Burgess, J. A.; Campanale, K. M.; Chirgadze, N. Y.; Clawson, D. K.; Dressman, B. A.; Hatch, S. D.; Khalil, D. A.; Kosa, M. B.; Lubbehusen, P. P.; Muesing, M. A.; Patick, A. K.; Reich, S. H.; Su, K. S.; Tatlock, J. H. J. Med. Chem. 1997, 40, 3979. Liu, G.; Huth, J. R.; Olejniczak, E. T.; Mendoza, R.; DeVries, P.; Leitza, S.; Reilly, E. B.; Okasinski, G. F.; Fesik, S. W.; von Geldern, T. W. J. Med. Chem. 2001, 44, 1202. Liu, L.; Stelmach, J. E.; Natarajan, S. R.; Chen, M.-H.; Singh, S. B.; Schwartz, C. D.; Fitzgerald, C. E.; O'Keefe, S. J.; Zaller, D. M.; Schmatz, D. M.; Doherty, J. B. Bioorg. Med. Chem. Lett. 2003, 13, 3979. Parnham, M. J.; Graf, E. Prog. Drug Res. 1991, 36, 9. Goudgaon, N. M.; Naguib, F. N. M.; el Kouni, M. H.; Schinazi, F. J. Med. Chem. 1993, 36, 4250. Mugesh, G.; du Mont, W.-W.; Sies, H. Chem. ReV. 2001, 101, 2125. Zhang, S.-J.; Dong, J.-Q.;Wang, Y.-G. Synth. Commun. 2003, 33, 1891.
} 
${ }^{2}$ Okamoto, Y., in Patai, S., Rappoport, Z., Eds. The Chemistry of Organic Selenium and Tellurium Compounds; Wiley: Chichester, U.K., 1986; Vol. 1, Chapter 10. Hellberg, J.; Remonen, T.; Johansson, M.; InganaÈs, O.; Theander, M.; Engman, L.; Eriksson, P. Synth. Met. 1997, 84, 251. Ando, T.; Kwon, T. S.; Kitagawa, A.; Tanemura, T.; Kondo, S.; Kunisada, H.; Yuki, Y. Macromol. Chem. Phys. 1996, 197, 2803. Takagi, K.; Nishikawa, Y.; Kwon, T. S.; Kunisada, H.; Yuki, Y. Polym. J. 2000, 32, 970. StuhrHansen, N.; Beckers, E. H. A.; Engman, L.; Janssen, R. A. J. Heteroat. Chem. 2005, 16, 656.

${ }^{3}$ Araujo, M.; Comasseto, J.V., Synlett 1995, 1145. Zeni, G.; Comasseto, J. V. Tetrahedron Lett. 1999, 40, 4619. Araujo, M.; Raminelli, C.; Comasseto, J. V.; Braz, J. Chem. Soc. 2004, 15, 358. Zeni, G.; Perin, G.; Cella, R.; Jacob, R. G.; Braga, A. L.; Silveira, C. C.; Stefani, H. A. Synlett 2002, 975. Vieira, M. L.; Zinn, F. K.; Comasseto, J. V.; Braz. J. Chem. Soc. 2001, 12, 586; Zeni, G.; Braga, A. L.; Stefani, H. A. Acc. Chem. Res. 2003, 36, 731.

${ }^{4}$ Nicolaou, K. C.; Dai, W.-M. Angew. Chem., Int. Ed. 1991, 30 , 1387.

${ }^{5}$ Uemura, S.; Wakasugi, M.; Okano, M. J. Organomet. Chem., 1980, 194, 277. Comasseto, J. V.; Stefani, H. A.; Chieffi , A.; Zukerman-Schpector J. Organometallics 1991, 10, 845-846.

6 “Tellurium in Organic Synthesis” $2^{\text {nd }}$ edition N. Petragnani, H. A. Stefani. Academic Press 2007.

${ }^{7}$ Cezar de Almeida, P.; dos Santos Tersariol, I. L.; Cezar de Oliveira, W. R.; Oliveira Rodrigues Cunha, R. L. Braz. Pat. BR 2005005808, 2007, CAN 148:231842, 2008

${ }^{8}$ Lenardaõ, E. J.; Silva, M. S.; Mendes, S. R.; de Azambuja, F.; Jacob, R. G.; dos Santos, P. C. S. Perin, G.; Braz. J. Chem. Soc., 2007, 18, 943-950. Perin, G.; Jacob, R. G.; Azambuja, F.; Botteselle, G. V.; Siqueira, G. M.; Freitaga, R. A.; Lenardaõ, E. J. Tetrahedron Lett. 2005, 46, 1679-1682. Perin, G.; Mendes, S. R.; Silva, M. S.; Lenardaõ, E. J.; Jacob, R. G.; dos Santos, P. C. Synth. Commun.2006, 36: 2587-2595. Kumar, S.; Engman, L. J. Org. Chem. 2006, 71, 5400-5403. Šibor, J.; Pazdera, P. Molecules 1996, 1, 157-162.

${ }^{9}$ Singh, K.; McWhinnie, W. R.; Chen, H. L.; Sun, M.; Hamor, T. A. J. Chem. Soc., Dalton Trans., 1996, 1545-1549. Ming Zheng', M.; Wang, L.; Shao, J.; Zhong, Q. Synth. Commun. 1997, 27, 351-354. Ericsson, C.; Engman, L. J. Org. Chem. 2004, 69, 5143-5146.

${ }^{10}$ Cunha, R. L. O. R.; Omori, A. T.; Castelani, P.; Toledo, F. T.; Comasseto, J. V. J. Organometallic Chem. 2004, 689, 3631-3636.

${ }^{11}$ Reichel, L.; Kirschbaum, E. Liebigs Ann. Chem. 1936, 523, 211. 